PROCEEDINGS OF THE

AMERICAN MATHEMATICAL SOCIETY

Volume 130, Number 6, Pages 1669-1672

S 0002-9939(01)06375-4

Article electronically published on October 12, 2001

\title{
A NOTE ON THE DISTANCE SET PROBLEM IN THE PLANE
}

\author{
THEMIS MITSIS
}

(Communicated by David Preiss)

\begin{abstract}
We use a simple geometric-combinatorial argument to establish a quantitative relation between the generalized Hausdorff measure of a set and its distance set, extending a result originally due to Falconer.
\end{abstract}

Let $A$ be a subset of the plane. The distance set $D(A)$ is defined as the set of all distances between points of $A$. Namely

$$
D(A)=\{|x-y|: x, y \in A\} .
$$

It follows from the work of Falconer [2] (see also Mattila [3]) that $\frac{1}{2} \leq \operatorname{dim}(A) \leq$ $\frac{3}{2} \Rightarrow \operatorname{dim}(D(A)) \geq \operatorname{dim}(A)-\frac{1}{2}$ and $\operatorname{dim}(A)>\frac{3}{2} \Rightarrow \mathcal{L}^{1}(D(A))>0$, where $\operatorname{dim}$ denotes Hausdorff dimension and $\mathcal{L}^{1}$ linear Lebesgue measure. This was improved by Wolff [4] who showed that $1<\operatorname{dim}(A) \leq \frac{4}{3} \Rightarrow \operatorname{dim}(D(A)) \geq \frac{3}{2} \operatorname{dim}(A)-1$ and $\operatorname{dim}(A)>\frac{4}{3} \Rightarrow \mathcal{L}^{1}(D(A))>0$. Putting these results together, one has the following estimate for the Hausdorff dimension of the distance set

$$
\operatorname{dim}(D(A)) \geq \begin{cases}\operatorname{dim}(A)-\frac{1}{2} & \text { if } \frac{1}{2} \leq \operatorname{dim}(A) \leq 1 \\ \frac{3}{2} \operatorname{dim}(A)-1 & \text { if } 1<\operatorname{dim}(A) \leq \frac{4}{3}\end{cases}
$$

It is a central open question in geometric measure theory whether $\operatorname{dim}(A)>1$ implies that $D(A)$ must have positive Lebesgue measure.

The proof of (1) involves techniques from harmonic analysis, more specifically, Bessel function estimates of the Fourier transform of radial functions and decay estimates for the $L^{2}$ circular means of the Fourier transform of a measure with finite energy. In this note we use a purely geometric argument to study the relation between a set and its distance set in terms of generalized Hausdorff measures. As a corollary, we obtain a sharpening of (1) in the case of sets of positive $s$-dimensional Hausdorff measure with $s<1$.

Before proceeding with the statement of our result we make some definitions.

$B(x, r)$ is the open disc of radius $r$ centered at $x$.

$Q(x, r)$ is the square of the form $[a, b) \times[a, b)$ with center at $x$ and diameter $r$.

If $A \subset \mathbb{R}^{2}$ is bounded, then its diameter is defined by $\operatorname{diam}(A)=\sup \{|x-y|$ : $x, y \in A\}$.

For a finite set $F, \operatorname{card}(F)$ denotes its cardinality.

Received by the editors November 21, 2000.

2000 Mathematics Subject Classification. Primary 28A12, 28A78.

This research has been supported by a Marie Curie Fellowship of the European Community programme "Improving human potential and the socio-economic knowledge base" under contract number HPMFCT-2000-00442. 
A measure function is a non-decreasing function $h(r), r \geq 0$, such that

$$
\lim _{r \rightarrow 0} h(r)=0 .
$$

The generalized Hausdorff outer measure $\Lambda_{h}$ with respect to a measure function $h$ is defined for $A \subset \mathbb{R}^{2}$ by

$$
\Lambda_{h}(A)=\operatorname{supinf}_{\delta>0}\left\{\sum_{j} h\left(r_{j}\right): A \subset \bigcup_{j} B\left(x_{j}, r_{j}\right), r_{j}<\delta\right\} .
$$

When $h(r)=r^{s}, \Lambda_{h}$ is the usual Hausdorff outer measure denoted by $\mathcal{H}^{s}$.

Our result is as follows.

Theorem. Let $C$ be a bounded Suslin subset of $\mathbb{R}^{2}$ and let $h$ be a measure function. Suppose that $\Lambda_{h}(C)>0$ and let

$$
\psi(r)=\left(h(r)\left(h(\operatorname{diam}(C))+\int_{1}^{\frac{1}{r}} h(1 / s) d s\right)\right)^{\frac{1}{2}} .
$$

Then $\Lambda_{\psi}(D(C))>0$ whenever $\psi$ makes sense as a measure function.

Proof. By Frostman's Lemma (see Carleson [1]), there exists a measure $\mu$ supported in $C$ such that $\mu(B(x, r)) \leq h(r) \forall x \in \operatorname{spt}(\mu)$. Let $a, b \in C$ be two points of density with respect to $\mu$. Choose $R>0$ such that $10 R<|a-b|$ and let

$$
A=B(a, R) \cap C, B=B(b, R) \cap C .
$$

Define

$$
D(A, B)=\{|x-y|: x \in A, y \in B\} .
$$

Let $\left\{\left(r_{k}, r_{k}+\epsilon_{k}\right)\right\}_{k}$ be a countable covering of $D(A, B)$. Without loss of generality, we may assume that $\frac{8}{10}|a-b| \leq r_{k} \leq \frac{12}{10}|a-b|$. Then

$$
\mu(A) \mu(B) \leq \sum_{k} \mu \times \mu\left\{(x, y) \in A \times B: r_{k}<|x-y|<r_{k}+\epsilon_{k}\right\} .
$$

Fix $k$ and let $\left\{Q\left(x_{i}, \epsilon_{k}\right)\right\}_{i \in I},\left\{Q\left(y_{j}, \epsilon_{k}\right)\right\}_{j \in J}$ be disjoint coverings of $A$ and $B$ respectively. Put $a_{i}=\mu\left(A \cap Q\left(x_{i}, \epsilon_{k}\right)\right), b_{j}=\mu\left(B \cap Q\left(y_{j}, \epsilon_{k}\right)\right)$ and define the following sets of indices:

$$
\begin{aligned}
\mathcal{I} & =\left\{(i, j) \in I \times J: r_{k}-2 \epsilon_{k}<\left|x_{i}-y_{j}\right|<r_{k}+3 \epsilon_{k}\right\}, \\
\mathcal{A}(j) & =\{i \in I:(i, j) \in \mathcal{I}\}, j \in J, \\
\mathcal{B}(i) & =\{j \in J:(i, j) \in \mathcal{I}\}, i \in I .
\end{aligned}
$$

Then, letting

$$
M_{k}=(\mu \times \mu)\left(\left\{(x, y) \in A \times B: r_{k}<|x-y|<r_{k}+\epsilon_{k}\right\}\right),
$$


we have

$$
\begin{aligned}
M_{k} & \leq \sum_{(i, j) \in \mathcal{I}} a_{i} b_{j} \\
& =\sum_{i \in I} a_{i} \sum_{j \in \mathcal{B}(i)} b_{j} \\
& \leq\left(\sum_{i \in I} a_{i}\right)^{\frac{1}{2}}\left(\sum_{i \in I} a_{i}\left(\sum_{j \in \mathcal{B}(i)} b_{j}\right)^{2}\right)^{\frac{1}{2}} \\
& =\mu(A)^{\frac{1}{2}}\left(\sum_{i \in I} a_{i} \sum_{j_{1}, j_{2} \in \mathcal{B}(i)} b_{j_{1}} b_{j_{2}}\right)^{\frac{1}{2}} \\
& =\mu(A)^{\frac{1}{2}}\left(\sum_{j_{1}, j_{2} \in J} b_{j_{1}} b_{j_{2}} \sum_{i \in \mathcal{A}\left(j_{1}\right) \cap \mathcal{A}\left(j_{2}\right)} a_{i}\right)^{\frac{1}{2}} .
\end{aligned}
$$

Now for fixed $j_{1}, j_{2} \in J$, the set $\left\{x_{i}: i \in \mathcal{A}\left(j_{1}\right) \cap \mathcal{A}\left(j_{2}\right)\right\}$ is contained in the intersection of the two annuli $\left\{x: r_{k}-2 \epsilon_{k}<\left|x-y_{j_{1}}\right|<r_{k}+3 \epsilon_{k}\right\},\left\{x: r_{k}-2 \epsilon_{k}<\right.$ $\left.\left|x-y_{j_{2}}\right|<r_{k}+3 \epsilon_{k}\right\}$. Using geometry we can show that the area of this intersection is at most

$$
\frac{100 r_{k}^{2} \epsilon_{k}^{2}}{\left|y_{j_{1}}-y_{j_{2}}\right| \sqrt{\left(2 r_{k}\right)^{2}-\left|y_{j_{1}}-y_{j_{2}}\right|^{2}}} \leq \frac{100\left(\frac{12}{10}|a-b|\right)^{2} \epsilon_{k}^{2}}{\left|y_{j_{1}}-y_{j_{2}}\right| \sqrt{(16 R)^{2}-(4 R)^{2}}} .
$$

Note that $\left\{x_{i}\right\}_{i \in I}$ and $\left\{y_{j}\right\}_{j \in J}$ are $\epsilon_{k}$-separated sets of points in the plane. Therefore, we conclude that

$$
\operatorname{card}\left(\left\{i \in \mathcal{A}\left(j_{1}\right) \cap \mathcal{A}\left(j_{2}\right)\right\}\right) \lesssim \frac{1}{\epsilon_{k}+\left|y_{j_{1}}-y_{j_{2}}\right|} .
$$

Furthermore, $a_{i} \leq h\left(\epsilon_{k}\right)$ because $Q\left(x_{i}, \epsilon_{k}\right) \subset B\left(x_{i}, \epsilon_{k}\right)$. It follows that

$$
\begin{aligned}
M_{k} & \lesssim \mu(A)^{\frac{1}{2}}\left(\sum_{j_{1}, j_{2} \in J} b_{j_{1}} b_{j_{2}} \frac{h\left(\epsilon_{k}\right)}{\epsilon_{k}+\left|y_{j_{1}}-y_{j_{2}}\right|}\right)^{\frac{1}{2}} \\
& =\mu(A)^{\frac{1}{2}} h\left(\epsilon_{k}\right)^{\frac{1}{2}}\left(\sum_{j_{1} \in J} b_{j_{1}} \sum_{j_{2} \in J} \frac{b_{j_{2}}}{\epsilon_{k}+\left|y_{j_{1}}-y_{j_{2}}\right|}\right)^{\frac{1}{2}} \\
& \left.\lesssim \mu(A)^{\frac{1}{2}} h\left(\epsilon_{k}\right)^{\frac{1}{2}}\left(\sum_{j_{1} \in J} b_{j_{1}} \sum_{j_{2} \in J} \int_{Q\left(y_{j_{2}}, \epsilon_{k}\right)} \frac{d \mu(y)}{\epsilon_{k}+\left|y_{j_{1}}-y\right|}\right)^{\frac{1}{2}}\right)^{\frac{1}{2}} \\
& =\mu(A)^{\frac{1}{2}} h\left(\epsilon_{k}\right)^{\frac{1}{2}}\left(\sum_{j_{1} \in J} b_{j_{1}} \int_{B} \frac{d \mu(y)}{\epsilon_{k}+\left|y_{j_{1}}-y\right|}\right)^{\frac{1}{2}} \\
& =\mu(A)^{\frac{1}{2}} h\left(\epsilon_{k}\right)^{\frac{1}{2}}\left(\sum_{j_{1} \in J} b_{j_{1}} \int_{0}^{\frac{1}{\epsilon_{k}}} \mu\left(\left\{y \in B: \epsilon_{k}+\left|y_{j_{1}}-y\right|<1 / s\right\}\right) d s\right)^{\frac{1}{2}}
\end{aligned}
$$


1672

$$
\begin{aligned}
& \leq \mu(A)^{\frac{1}{2}} h\left(\epsilon_{k}\right)^{\frac{1}{2}}\left(\sum_{j_{1} \in J} b_{j_{1}}\left(\int_{0}^{1} \mu\left(B\left(y_{j_{1}}, 1 / s\right)\right) d s+\int_{1}^{\frac{1}{\epsilon_{k}}} h(1 / s) d s\right)\right)^{\frac{1}{2}} \\
& \leq \mu(A)^{\frac{1}{2}} h\left(\epsilon_{k}\right)^{\frac{1}{2}}\left(\sum_{j_{1} \in J} b_{j_{1}}\left(h(\operatorname{diam}(C))+\int_{1}^{\frac{1}{\epsilon_{k}}} h(1 / s) d s\right)\right)^{\frac{1}{2}} \\
& =\mu(A)^{\frac{1}{2}} \mu(B)^{\frac{1}{2}} \psi\left(\epsilon_{k}\right) .
\end{aligned}
$$

Hence, by (21)

$$
\mu(A)^{\frac{1}{2}} \mu(B)^{\frac{1}{2}} \lesssim \sum_{k} \psi\left(\epsilon_{k}\right) .
$$

This implies $\Lambda_{\psi}(D(C)) \geq \Lambda_{\psi}(D(A, B))>0$.

Taking $h(r)=r^{s}, \frac{1}{2}<s<1$, gives $\psi(r) \lesssim r^{s-\frac{1}{2}}$. Hence we obtain the following.

Corollary. Let $A$ be a subset of the plane such that $\mathcal{H}^{s}(A)>0$, with $\frac{1}{2}<s<1$. Then $\mathcal{H}^{s-\frac{1}{2}}(D(A))>0$.

Note that since an $\alpha$-dimensional set may have zero $\mathcal{H}^{\alpha}$-measure, our corollary extends (1) in the indicated range of dimensions.

\section{REFERENCES}

[1] Carleson, L. Selected problems on exceptional sets, Van Nostrand Math. Studies 13, Van Nostrand, Princeton, N.J., 1967. MR 37:1576

[2] Falconer, K.J. On the Hausdorff dimension of distance sets, Mathematika 32 (1985), 206-212. MR 87j:28008

[3] Mattila, P. Spherical averages of Fourier transforms of measures with finite energy; dimension of intersections and distance sets, Mathematika 34 (1987), 207-228. MR 90a:42009

[4] Wolff, T. Decay of circular means of Fourier transforms of measures, Internat. Math. Res. Notices (1999), 547-567. MR 2000k:42016

Department of Mathematics and Statistics, University of Jyväskylä, P.O. Box 35, FIN-40351 JYVÄSKYLÄ, FinLAND

E-mail address: mitsis@math.jyu.fi

Current address: Nestou 6, Athens 14342, Greece

E-mail address: tmitsis@yahoo.com 